\title{
Research Article \\ Hsa_circ_0008309 May Be a Potential Biomarker for Oral Squamous Cell Carcinoma
}

\author{
Bowen Li, ${ }^{1,2}$ Feng Wang, ${ }^{1}$ Xiang Li, ${ }^{1}$ Shuai Sun, ${ }^{1}$ Yuehong Shen $\mathbb{D}^{1},{ }^{1}$ and Hongyu Yang $\mathbb{D}^{1}$ \\ ${ }^{1}$ Department of Oral and Maxillofacial Surgery, Peking University Shenzhen Hospital, Shenzhen, Guangdong 518036, China \\ ${ }^{2}$ Department of Oral and Maxillofacial Surgery, Sun Yat-sen Memorial Hospital, Sun Yat-sen University, Guangzhou 510120, China
}

Correspondence should be addressed to Yuehong Shen; yuehongshen@hotmail.com and Hongyu Yang; hyyang192@hotmail.com

Received 11 April 2018; Revised 2 August 2018; Accepted 12 August 2018; Published 23 September 2018

Academic Editor: Michalis V. Karamouzis

Copyright (C) 2018 Bowen Li et al. This is an open access article distributed under the Creative Commons Attribution License, which permits unrestricted use, distribution, and reproduction in any medium, provided the original work is properly cited.

\begin{abstract}
Objective. Oral squamous cell carcinoma (OSCC) is the most common cancer of the head and neck region. The circular RNA (circRNA) is known to serve an important role in the carcinogenesis of different types of cancer. However, the circRNA role of OSCC remains unclear. Methods. 8 pairs of OSCC tissues and adjacent normal tissues were obtained to detect circRNAs expression by high-throughput sequencing, and 45 pairs of OSCC tissues were selected to verify the differentially significant circRNAs by reverse transcription-quantitative polymerase chain reaction (RT-qPCR). To further investigate the role of hsa_circ_0008309, the circRNA-microRNA (miR)-mRNA network was predicted using bioinformatics databases. The expression levels of hsa_circ_0008309, miR-1290, miR-136-5P, and miR-382-5P in SCC-15 and CAL27 cell lines were detected by RT-qPCR. Western blotting was performed to detect the protein level of Ataxin 1 (ATXN1). Results. The highthroughput sequencing results demonstrated that circRNAs were abundantly expressed in OSCC, and 16 circRNAs were significantly differentially expressed. Hsa_circ_0008309 was significantly downregulated in 45 pairs of OSCC tissue samples and was statistically correlated with pathological differentiation. The bioinformatics databases suggested that hsa_circ_0008309 could combine with miR-1290, miR-136-5P, and miR-382-5P, respectively, to regulate the expression of ATXN1. It was subsequently identified that hsa_circ_0008309 may inhibit miR-136-5P and miR-382-5P expression and increase ATXN1 expression in the OSCC cell lines. Conclusion. In summary, the results of the present study revealed that OSCC tissues have abundant circRNAs and, to the best of our knowledge, we firstly explore the regulatory role of the hsa_circ_0008309-miR136-5P/hsa-miR-382-5P-ATXN1 network in OSCC. The results indicated that hsa_circ_0008309 may be a potential biomarker for OSCC.
\end{abstract}

\section{Introduction}

Oral squamous cell carcinoma (OSCC), the most frequently occurring oral malignancy, is the sixth most prevalent cancer worldwide and the third most common carcinoma in some developing countries $[1,2]$. OSCC is one of the most frequently occurring head-neck tumors and accounts for $\sim 80 \%$ of all cases [3]. In addition, $\sim 600,000$ new clinical cases are diagnosed annually and the age at the time of diagnosis is becoming lower each year [4]. Although the treatments modalities have improved, the mortality rate has not decreased significantly and the 5-year survival rate of OSCC is only 63\% [5]. Cancers including OSCC have been widely regarded as gene-related diseases; however, the precise molecular mechanisms and genetic basis of OSCC carcinogenesis remain largely unclear. Therefore, it is imperative to identify therapeutic targets to improve OSCC diagnosis and treatment.

Recently, circular RNAs (circRNAs) have been considered to be a special type of noncoding RNA, which are widespread and diverse in mammals [6]. Unlike linear RNA, circRNA contains covalently closed loop structures and has neither 5'caps nor 3'tails; therefore, the majority of circRNA cannot be degraded by RNA exonucleases or RNase R, thereby maintaining an improved stability compared with linear transcripts [6]. Emerging evidence has revealed various functions of circRNA such as competing endogenous RNAs, miRNA sponges, or transcriptional regulators [7]. CircRNA 
has also been considered to serve a crucial role in cancers, and due to its functions, circRNAs may serve as a potential target for tumor therapy or diagnostic biomarkers [8]. For example, CDRlas and circ-FOXO3 sponge microRNA (miR) regulate the development of breast carcinoma; additionally, circ-ITCH has an inhibitory effect on esophageal squamous cell carcinoma by suppressing the $\mathrm{Wnt} / \beta$-catenin pathway [9-11]. However, the role of circRNA in the initiation and progression of OSCC has not been completely elucidated. In the present study, circRNA expression profiles were analyzed through high-throughput sequencing, followed by RT-qPCR to verify the significantly expressed circRNAs in the OSCC tissues. Hsa_circ_0008309 was identified to be downregulated in the cancer tissues. In order to explore the functions of hsa_circ_0008309, the hsa_circ_0008309 miR binding sites were predicted, and closely associated circRNA-miR-mRNA networks were constructed using bioinformatics approaches. These networks were further investigated in OSCC cell lines. In summary, the data provided a novel basis for circRNA functional research in OSCC and indicated that hsa_circ_0008309 may be a novel potential biomarker in OSCC research.

\section{Materials and Methods}

2.1. Patients and Samples. A total of 45 pairs of frozen OSCC tissues and adjacent normal tissues were acquired from patients with OSCC and these tissues were prepared for high-throughput sequencing and validation by RT-qPCR. These samples were acquired from the Department of Oral and Maxillofacial Surgery of Peking University Shenzhen Hospital (Shenzhen, China) from June 2015 to June 2016. Patients had not undergone additional treatments prior to surgery and all OSCC tissues were confirmed by strict pathological examination. The age of patients ranged from 2978 years, and the median age at the time of diagnosis was 54 years. The male to female ratio was $34: 11$. Tissues were obtained from the tongue, gingiva, bucca, and floor of the mouth. Clinical and pathological characteristics of patients were based on the most recent World Health Organization (WHO) classification and UICC tumor-node-metastasis (TNM) classification [12]. Written informed consent from all patients was obtained, and the study was approved by the Medical Ethics Committee of Peking University Shenzhen Hospital. All methods were performed in accordance with the relevant guidelines and regulations.

2.2. RNA Extraction. Samples obtained from surgical specimens were immediately frozen using liquid nitrogen. Total RNA was extracted from frozen tissues using TRIzol ${ }^{\circledR}$ reagent (Thermo Fisher Scientific, Inc., Waltham, MA, USA) according to the manufacturer's protocol. The quality and quantity of the RNA were evaluated at a 260/280 ratio using a NanoDrop spectrophotometer (Thermo Fisher Scientific, Inc.).

\subsection{Library Construction and High-Throughput Sequencing.} Following extraction, total RNA was treated with RNase $\mathrm{R}$ to degrade the linear RNA and purified with RNeasy
MinElute Cleanup Kit (Qiagen, Inc., Valencia, CA, USA). Next, a strand-specific library was constructed with VAHTS Total RNA-seq (H/M/R) Library Prep Kit for Illumina according to the manufacturer's protocol. In brief, ribosomal RNA was removed to retain the circRNAs. The enriched circRNAs were broken into short fragments using a fragmentation buffer and reverse transcribed into cDNA with random primers. Secondly, strand cDNA fragments synthesized by DNA polymerase I were purified with VAHTSTM DNA Clean Beads and liquated to Illumina sequencing adapters. Uracil-N-glycosylase was used to digest the secondstrand cDNA. The digested products were purified with VAHTSTM DNA Clean Beads, amplified, and sequenced with Illumina HiSeq ${ }^{\mathrm{TM}} 2500$ by Gene Denovo Biotechnology Co. (Guangzhou, China). The edgeR package (http://www. rproject.org/) was used to identify differentially expressed circRNAs. Some significant circRNAs were blasted against the circBase for annotation [13]. The circRNAs that could not be annotated were defined as novel circRNAs.

2.4. $R T$ - $q P C R$. The reaction mixture $(20 \mu \mathrm{l})$ containing $1 \mu \mathrm{g}$ of total RNA was reverse transcribed into cDNA with the PrimeScript RT Master Mix (Takara Biotechnology Co., Ltd., Dalian, China). The mixture was incubated at $37^{\circ} \mathrm{C}$ for $15 \mathrm{~min}$ and $85^{\circ} \mathrm{C}$ for $5 \mathrm{sec}$ to acquire cDNA. RT-qPCR was conducted with a Roche Applied Science LightCycler ${ }^{\circledR} 96$ Real-Time PCR System (Roche Diagnostics, Indianapolis, Indiana, USA) in accordance with the manufacturer's protocol. The reaction mixture comprised of $2 \mu \mathrm{l} \mathrm{cDNA}, 5 \mu \mathrm{l}$ SYBR $^{\circledR}$ Premix Ex Taq ${ }^{\mathrm{TM}}$ II (Takara, China) and $1 \mu$ l primers (reverse and forward) with RNase-Free water to a final volume of $10 \mu \mathrm{l}$. The thermocycling conditions were as follows: $95^{\circ} \mathrm{C}$ for $3 \mathrm{~min}$ and amplified by 40 cycles of denaturing at $95^{\circ} \mathrm{C}$ for $10 \mathrm{sec}$ and $60^{\circ} \mathrm{C}$ for $30 \mathrm{sec}$. $\beta$-actin was used as an internal standard. Melting curves were produced to check product purity and the expression levels of circRNAs were detected by using the $2^{-\Delta \Delta \mathrm{Cq}}$ method. The $\mathrm{Cq}$ value was the fractional cycle number at which the fluorescence exceeded the given threshold [14]. Primer sequences are listed as follows: hsa_circ_0008309 forward primer: $5^{\prime}$-AC AGCTATGGTGATGATTAGAGACA-3'; hsa_circ_0008309 reverse primer: $5^{\prime}$-TCAGAAGGTCCCAAATGCTGTT-3'; $\beta$-actin forward primer: $5^{\prime}$-AAACTGGAACGGTGAAGG TG-3'; $\beta$-actin reverse primer: $5^{\prime}$-AGTGGGGTGGCTTT TAGGAT- $3^{\prime}$.

2.5. Prediction for the CircRNA-miRNA-mRNA Interaction. To investigate hsa_circ_0008309 function, the circRNAmiR-mRNA network was theoretically predicted through DIANA (http://diana.imis.athena-innovation.gr), MiRanda (http://www.microrna.org), and TargetScan (http://www. targetscan.org) databases. According to conserved seedmatching sequence principles, the predicted miRs and potential target genes were chosen by identifying the intersection of three databases [15]. The graph of the circRNA-miRmRNA network was visualized by Cytoscape 3.01 (http:// www.cytoscape.org). 
2.6. Vector Construction. In order to produce the hsa_circ_0008309 transcript formation in vitro by nonlinear splicing, we constructed the hsa_circ_0008309 overexpression vector. The hsa_circ_0008309 linear sequence was synthesized and added to the pLCDH-ciR vector (Geneseed Biotechnology Co., Guangzhou, China) for the circularization of transcripts. The front circular frame contained the endogenous flBiotec genomic sequence with an EcoRI restriction enzyme site, and the back circular frame contained part of the inverted upstream sequence with a BamHI site. The cDNA encoding hsa_circ_0008309 was amplified using primers $5^{\prime}$-CGGAATTCTGAAATATGCTATCTTACAGA TGACCATGGATGAAAAATATGTA-3' ${ }^{\prime}$ and $5^{\prime}$-CGGGAT CCTCAAGAAAAAATATATTCACCATGTACATTAGTAT GTCTCTA-3' in the 293T cell line (Geneseed Biotechnology Co.). As a result, the amplified fragment was cloned into the vector between the two reading frames, and the mock vector was confirmed to contain a nonsense sequence between the two circular frames without the hsa_circ_0008309 encoding cDNA. The result of vector construction was verified by direct sequencing. The vectors were constructed with the help of Guangzhou Geenseed Biotech Co.

2.7. Cell Culture and Plasmid Transfection. The 293T cell line (Geneseed Biotechnology Co.) and SCC15 and CAL27 (purchased from the American Type Culture Collection, Manassas, VA, USA) cell lines were cultured in Dulbecco's modified Eagle's medium supplemented with $10 \%$ fetal bovine serum (both from Gibco; Thermo Fisher Scientific, Inc.), and culture plates were incubated at $37^{\circ} \mathrm{C}$ in a $5 \%$ $\mathrm{CO}_{2}$ humidified incubator. The empty vector without the hsa_circ_0008309 encoding cDNA was used as negative control. According to the manufacturer's protocol, hsa_circ_0008309 overexpression vector, miR-136-5P inhibitor plus hsa_circ_0008309 expression plasmid, miR-382-5P inhibitor plus hsa_circ_0008309 expression plasmid, and an empty control vector was, respectively, transfected into cell lines with $4000 \mathrm{ng}$ plasmids using Lipofectamine 3000 (Invitrogen; Thermo Fisher Scientific, Inc.), and cell lines were harvested at $24 \mathrm{~h}$ following transfection.

2.8. Western Blotting. Cells were lysed with Blue Loading Buffer Pack (Cell Signaling Technology, Inc.) with a protease inhibitor cocktail and phenylmethanesulfonyl fluoride (Cell Signaling Technology, Inc.). The protein concentrations were quantified using a BCA Protein Assay Kit (Cell Signaling Technology, Inc.). Total cellular proteins $(20 \mu \mathrm{g})$ were separated via $10 \%$ SDS-PAGE and transferred to a polyvinylidene difluoride membrane (Millipore). Bovine serum albumin (BSA; 5\%) was used as a blocking agent to reduce background and nonspecific binding. After blocking for $1 \mathrm{~h}$, the membranes were incubated overnight at $4^{\circ} \mathrm{C}$ with rabbit monoclonal anti-human Ataxin 1 (ATXN1) and rabbit monoclonal anti-human GAPDH (both 1:1000; both from Cell Signaling Technology, Inc.) antibodies. GAPDH was used as a loading control for normalization. Following intensive washing, the membranes were incubated with anti-rabbit horseradish peroxidase-conjugated secondary antibodies ( $1: 1000$, Cell Signaling Technology, Inc.) for $1 \mathrm{~h}$ at room

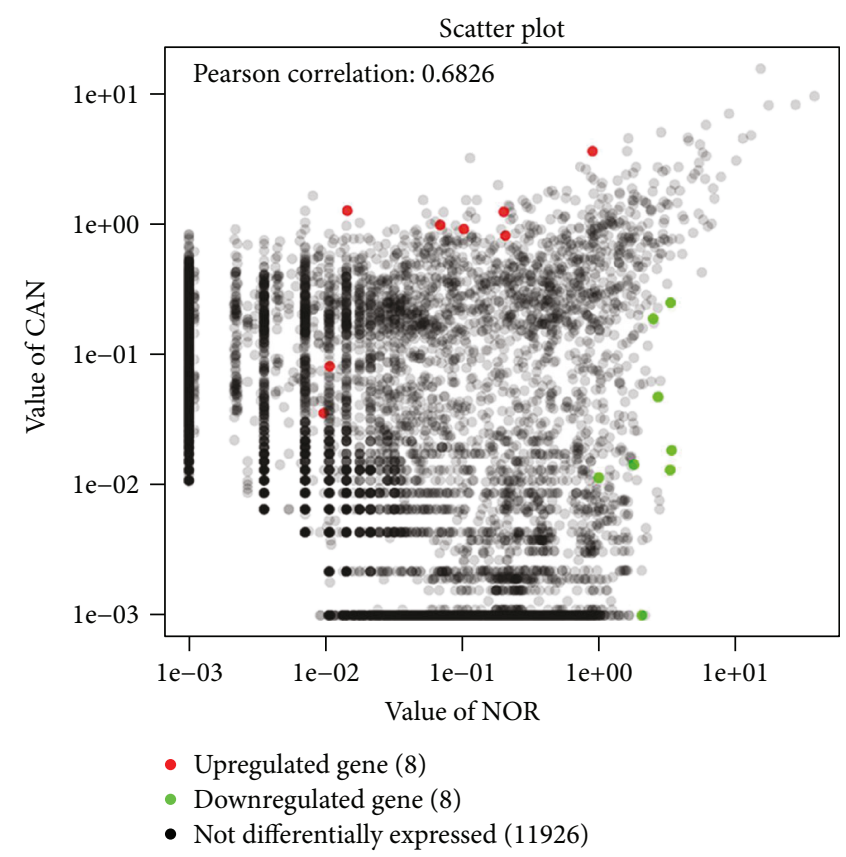

FIgURE 1: Scatter plot illustrating the expression profile of 11,942 circRNAs between the OSCC tissue and adjacent normal tissue. The horizontal lines represent circRNAs expression in the adjacent normal tissues and vertical lines represent circRNAs expression in OSCC tissues. The red plots represent significantly upregulated circRNAs in OSCC tissues, and green plots represent significantly downregulated circRNAs in OSCC tissues. NOR: normal tissues; CAN: cancer tissues; OSCC: oral squamous cell carcinoma; circRNA: circular RNA.

temperature. The protein bands were visualized using SignalFire $^{\mathrm{TM}}$ ECL Reagent with the ImageQuant LAS4000 system (Fujifilm, Tokyo, Japan).

2.9. Statistical Analysis. All experiments were repeated three times, and data were presented as the means \pm standard deviation. Differences and characterizations in circRNA expression profiles between OSCC tissues and adjacent noncarcinoma tissues were assessed by Pearson's correlation test. Hsa_circ_0008309 expression level between OSCC tissues and para-cancerous tissues was evaluated by two-tailed Student's $t$-test. The Student's $t$-test (two-tailed) was performed to analyze the association between the hsa_circ_0008309 expression level and the clinicopathological features of patients with OSCC. A one-way analysis of variance test was used to analyze hsa_circ_0008309 expression between different pathological groups, and the post hoc test was Tukey's multiple comparison test. Correlations between the circRNA expression level and miRNAs were evaluated by one-way ANOVA and Tukey's multiple comparison test. The clinical diagnostic value of hsa_circ_0008309 was verified by receiver operating characteristic (ROC) curve analysis in which an area under the curve $(\mathrm{AUC})=0.5$ indicated no diagnostic value. $P<0.05$ was considered to indicate a statistically significant difference. All statistical analyses were performed by GraphPad Prism 5.0 (GraphPad Software, La Jolla, CA, USA). 
TABLE 1: 16 differently expressed circRNAs in the oral squamous cell carcinoma.

\begin{tabular}{|c|c|c|c|c|c|}
\hline CircRNA & Fold change & $P$ value & Type & chr & Gene Symbol \\
\hline Hsa_circ_0008202 & -8.00076 & 0.042153 & Exons & 1 & SPATA6 \\
\hline Hsa_circ_0004491 & -3.73247 & 0.022494 & Exons & 2 & ORC4 \\
\hline Hsa_circ_0008309 & 2.010811 & 0.023438 & Exons & 2 & CUL3 \\
\hline Novel_circ_006041 & -11.0136 & 0.035522 & Exons & 2 & FANCL \\
\hline Novel_circ_007300 & -7.53215 & 0.042153 & One_exon & 22 & $\mathrm{MB}$ \\
\hline Novel_circ_007366 & -6.97964 & 0.036032 & Exons & 22 & FBLN1 \\
\hline Novel_circ_009346 & -6.46152 & 0.042153 & Exons & 5 & EBF1 \\
\hline Novel_circ_006206 & -5.84394 & 0.042153 & Exons & 2 & RMND5A \\
\hline Novel_circ_008049 & -3.74847 & 0.034611 & Exons & 3 & PHC3 \\
\hline Novel_circ_002578 & 1.872629 & 0.030545 & Antisense & 12 & KRT6C \\
\hline Novel_circ_005748 & 1.977281 & 0.035522 & Exons & 2 & MBOAT2 \\
\hline Novel_circ_009749 & 2.620575 & 0.036032 & Exons & 6 & PHIP \\
\hline Novel_circ_003644 & 2.918808 & 0.049827 & Intergenic & 14 & NA \\
\hline Novel_circ_007992 & 3.151974 & 0.036032 & Exons & 3 & RNF13 \\
\hline Novel_circ_010219 & 3.832216 & 0.035522 & Exons & 7 & FAM126A \\
\hline Novel_circ_003819 & 6.455806 & 0.036032 & Exons & 15 & SPPL2A \\
\hline
\end{tabular}

\section{Results}

3.1. Profile of Differentially Expressed CircRNAs in Patients with OSCC. A total of 11,942 circRNA targets, including 1921 known circRNAs and 10,021 novel circRNAs, were detected and defined in 8 pairs of OSCC samples and adjacent normal tissues through high-throughput sequencing (Figure 1). A total of 16 significantly different circRNAs were identified in 8 pairs of samples via high-throughput sequencing, and details regarding these circRNAs are presented in Table 1.

3.2. Validation of Hsa_circ_0008309 Expression in the OSCC Tissues. The 16 significantly differentially expressed circRNAs in 45 pairs of OSCC samples were analyzed by RT-qPCR. The results demonstrated that hsa_circ_0008309 was significantly downregulated in the carcinoma tissues (Figure 2(a)). According to the human reference genome (GRCh37/hg19) from the Ensembl genome database (http://www.ensembl.org), hsa_circ_0008309 is located at chr2: 225400244-225422573 and the parental gene is Cullin 3 (CUL3). The whole length of the CUL3 gene is $22,329 \mathrm{bp}$, while the mature transcript of the hsa_circ_0008309 is 312 bp.

3.3. The Relationship between Hsa_circ_0008309 and Clinicopathological Characteristics. To confirm the potential diagnostic value of hsa_circ_0008309, the clinicopathological characteristics of the OSCC patients were analyzed with respect to the hsa_circ_0008309 expression level in Table 2. The results found that hsa_circ_0008309 expression was significantly associated with pathological differentiation of OSCC patients. In addition, the diagnostic effect of hsa circ_0008309 was analyzed via the ROC curve. The area under the ROC curve was 0.7642, indicating the hsa circ_0008309 is relatively closely associated with OSCC (Figure 2(b)). Taken together, these data indicate that hsa_circ_0008309 may serve as a potential biomarker for the diagnosis of OSCC.

3.4. CircRNA-miR-mRNA Network Construction. Results from the bioinformatics analysis found that hsa circ_0008309 could, respectively, combine with miR-1290, miR-136-5P, and miR-382-5P (Figure 3(a)). A large number of target genes were identified and some were closely associated with more than one network, as indicated by the bioinformatics analysis (Figure 3(b)). The results demonstrated that ATXN1 was the only gene to be associated with all networks. Therefore, we hypothesize that ATXN1 may be regulated by hsa_circ_0008309 in OSCC.

3.5. Validation of Hsa_circ_0008309-miR-136-5P/miR-3825P-ATXN1 Pathway. After transfection, hsa_circ_0008309 was identified to be overexpressed in the SCC15 and CAL27 cell lines (Figure 4(a)). Compared with the negative control, miR-136-5P and miR-382-5P expression was downregulated in the hsa_circ_0008309 overexpression groups in the SCC15 and CAL27 cell lines (Figure 4(b)). Western blot analysis demonstrated that the ATXN1 protein level was increased when hsa_circ_0008309 expression was upregulated in SCC15 and CAL27 cell lines (Figure 4(c)). These results suggest that hsa_circ_0008309 may regulate the hsa_ circ_0008309-miR-136-5P/miR-382-5P-ATXN1 pathway in OSCC cell lines.

\section{Discussion}

CircRNA was previously considered to be a noise from aberrant RNA splicing [16-18]. However, it was recently identified to have the potential as miR sponges and ideal biomarkers in various diseases [19]. In addition, a large number of studies have indicated that various circRNAs were associated with the initiation and progression of cancers 


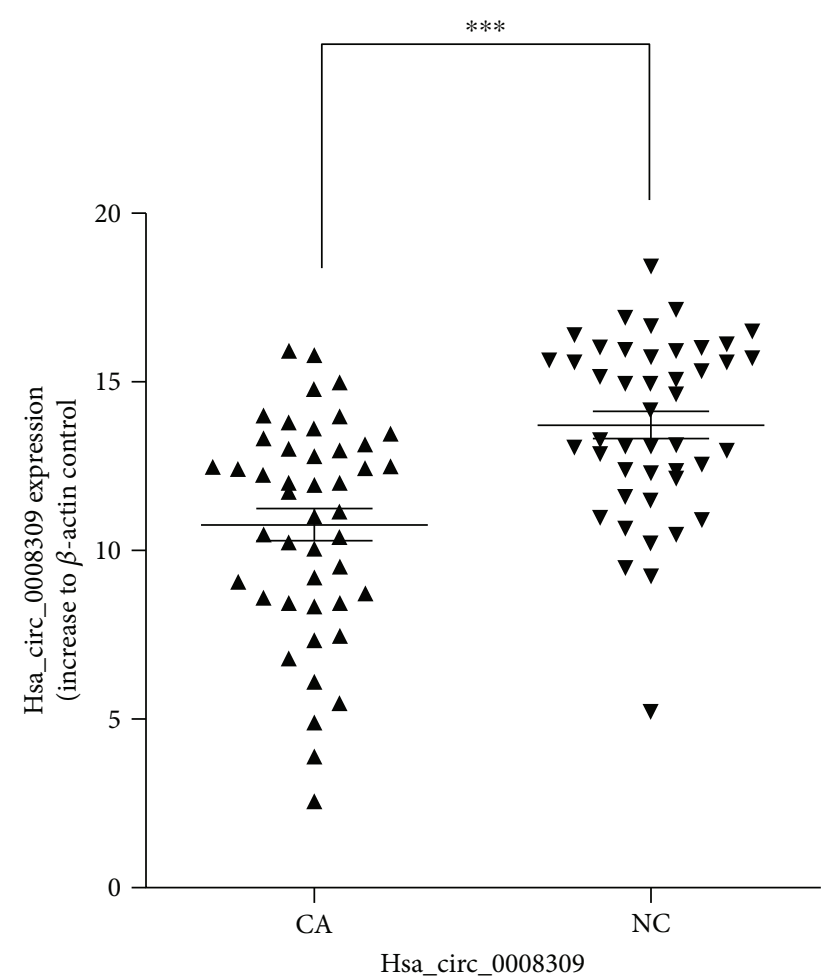

(a)

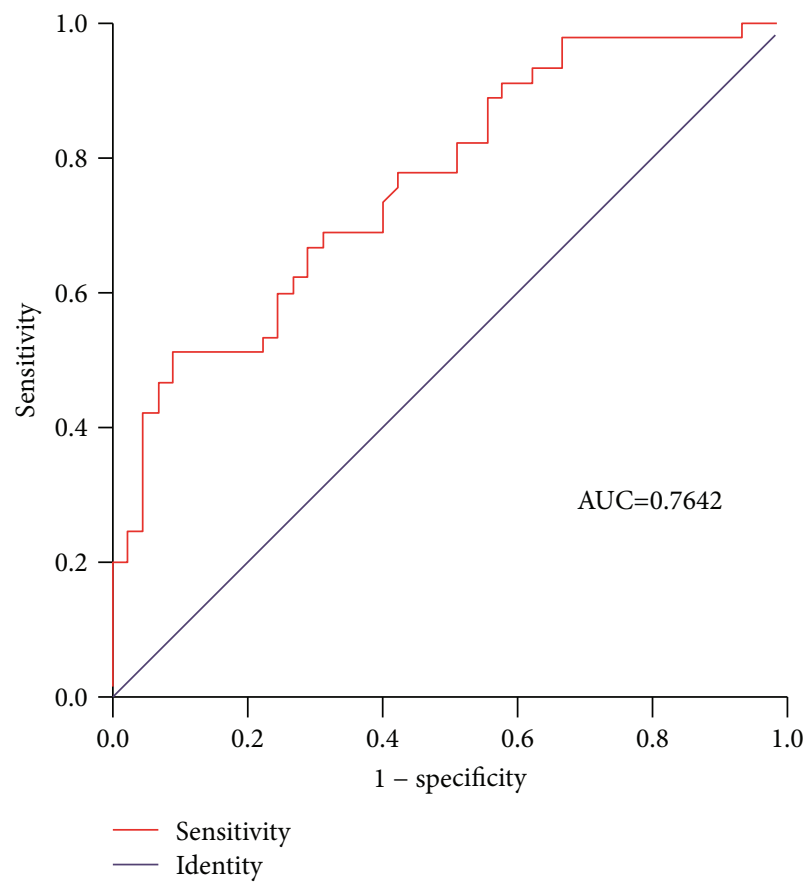

(b)

FIGURE 2: (a) Expression level of hsa_circ_0008309 validated by RTqPCR in OSCC tissues $(n=45)$ with adjacent normal tissue $(n=45)$. Student's $t$-test (two-tailed) was performed. Data are shown as the mean \pm standard deviation. (b) ROC analysis of the expression hsa_circ_0008309 in 45 paired OSCC patients. OSCC: oral squamous cell carcinoma; RT-qPCR: reverse transcriptionquantitative polymerase chain reaction; NC: normal tissues; $\mathrm{CA}$ : cancer tissues; ROC: receiver-operating characteristic; AUC: area under curve.
TABLE 2: Correlation between hsa_circ_0008309 expression and clinicopathological characteristics in 45 oral squamous cell carcinoma patients.

\begin{tabular}{|c|c|c|}
\hline Characteristics & Total cases $(n=45)$ & $P$ value \\
\hline Sex & & 0.9828 \\
\hline Male & 34 & \\
\hline Female & 11 & \\
\hline Age (years) & & 0.8206 \\
\hline Range (median) & $29-78(54)$ & \\
\hline$<60$ & $29(46.655)$ & \\
\hline$\geq 60$ & $16(66.125)$ & \\
\hline Site & & 0.2198 \\
\hline Tongue & 25 & \\
\hline Buccal & 8 & \\
\hline Gingiva & 5 & \\
\hline Floor of the mouth & 7 & \\
\hline Clinical stage & & 0.2682 \\
\hline 0\&I + II & 19 & \\
\hline III + IV & 26 & \\
\hline Pathological differentiation & & $0.0386^{*}$ \\
\hline Well & 19 & \\
\hline Moderately & 20 & \\
\hline Poorly & 6 & \\
\hline LNM & & 0.3176 \\
\hline Yes & 17 & \\
\hline No & 28 & \\
\hline
\end{tabular}

[20]; however, there is little evidence regarding the role of circRNA in OSCC.

In the present study, a total of 11,942 circRNAs were identified, demonstrating that circRNAs were in abundant existence in the OSCC samples $(n=8)$. Furthermore, 16 circRNAs were identified to be significantly differentially expressed in the OSCC samples through bioinformatics analysis. The results suggest that circRNA may serve an important role in OSCC, and these significantly differentially expressed circRNAs were subsequently validated in the 45 pairs of OSCC samples. As a result, hsa_circ_0008309 was demonstrated to be significantly downregulated in OSCC tissues $(P<0.001)$. Notably, compared with normal tissues, the hsa_circ_0008309 expression level was revealed to be 2.010811 times in OSCC tissues by high-throughput sequencing. The levels of hsa_circ_0008309 in certain samples were upregulated in the 45 pairs of OSCC tissues; however, the levels of hsa_circ_0008309 in the majority of samples were downregulated. These results indicated that hsa_circ_0008309 expression level of each patient was not consistent and varied between individuals. The expression of hsa_circ_0008309 was downregulated in tumor tissues and was, therefore, more likely to act as a tumor suppressor. The ROC analysis indicated that the hsa_circ_0008309 expression level exhibited a diagnostic role in distinguishing OSCC tissues from adjacent normal tissues. Taken together, 


$\begin{array}{lcc}\text { CircRNA-miRNA ID } & \text { CircRNA (top)-miRNA (bottom) pairing } & \text { Site type } \\ \text { hsa_circ_0008309 }\left(5^{\prime} \ldots 3^{\prime}\right) & \text { UGGUUUUGCAUAAACAUGGAGAA } & 7 \text { mer-1a } \\ \text { hsa-miR-136-5p }\left(3^{\prime} \ldots 5^{\prime}\right) & \text { AGGUAGUAGUUUUGUUUA I I I I } & \\ \text { hsa_circ_0008309 }\left(5^{\prime} \ldots 3^{\prime}\right) & \text { AAAAUGCAAUUCAAGAAA UCC AG } & 7 \text { mer-m8 } \\ \text { hsa-miR-1290 }\left(3^{\prime} \ldots 5^{\prime}\right) & \text { AGGGACUAGGUUUUU A GGU } & \\ \text { hsa_circ_0008309 }\left(5^{\prime} \ldots 3^{\prime}\right) & \text { UAAAUUCAUUGAAUACAACUUU } & 7 \text { mer-m8 } \\ \text { hsa-miR-382-5p }\left(3^{\prime} \ldots 5^{\prime}\right) & \text { GCUUAGGUGGUGCUUGUUGAG } & \end{array}$

(a)

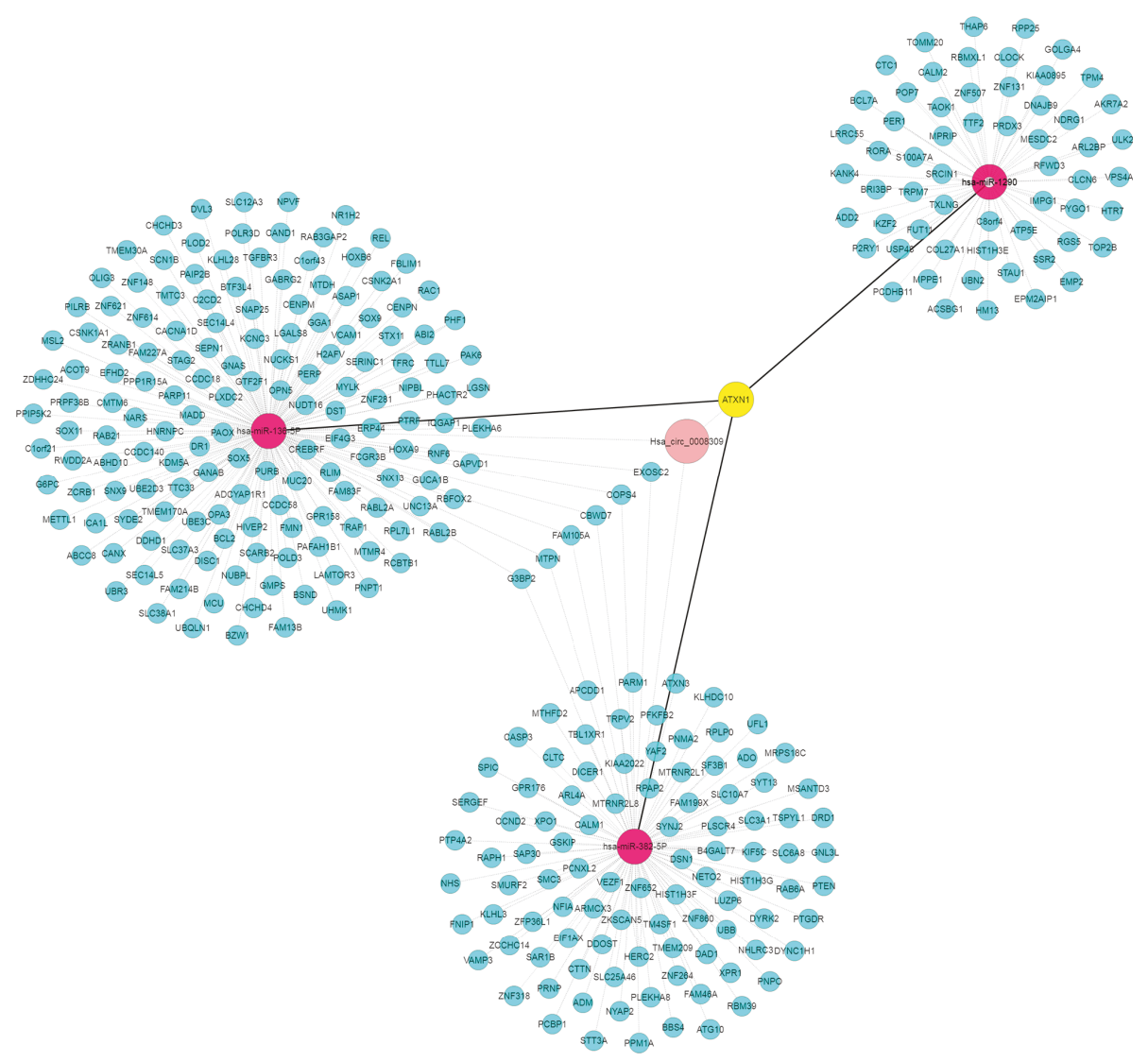

(b)

Figure 3: (a) Prediction of hsa_circ_0008309 and miRNAs interactions. (b) CircRNA-miRNA-mRNA network of hsa_circ_0008309. Circ: circular; mi: micro.

we hypothesized that hsa_circ_0008309 has a potential effect on OSCC.

Compared with linear RNA, circRNA has been reported to have an increased number of miR binding sites and may regulate gene expression by acting as miR sponges, thereby regulating linear RNA transcription and protein production [20]. Consequently, the present study examined whether hsa_circ_0008309 had the potential to affect the miRs in OSCC. The bioinformatics analysis illustrated that hsa_circ_0008309 could potentially interact with important miRs including miR-1290, miR-136-5P, and miR-382-5P. In addition, the ATXN1 gene was identified to be strongly associated with these miRs. Therefore, we speculated that hsa_circ_0008309 may regulate the ATXN1 gene by acting as a sponge to these miRs. In order to further explore the role of hsa_circ_0008309, hsa_circ_0008309 was overexpressed in SCC15 and CAL27 cell lines and miR-136-5P and miR382-5P expression were inhibited, and the ATXN1 protein level was increased when hsa_circ_0008309 was upregulated. ATXN1 was found predominantly in the nuclei of neurons and may function as a transcriptional regulator [21]. Moreover, ATXN1 is a component of the Notch signaling pathway [22], and the Notch signaling reportedly mediates tumor cell migration and invasion induced by low oxygen supply (hypoxia), which is a critical characteristic of solid tumors [23]. Previous studies have demonstrated that a potential link between ATXN1 expression (both upregulation and downregulation) and cancer development in humans could regulate cell proliferation and the epithelialmesenchymal transition of cells in various cancers [24, 25], 

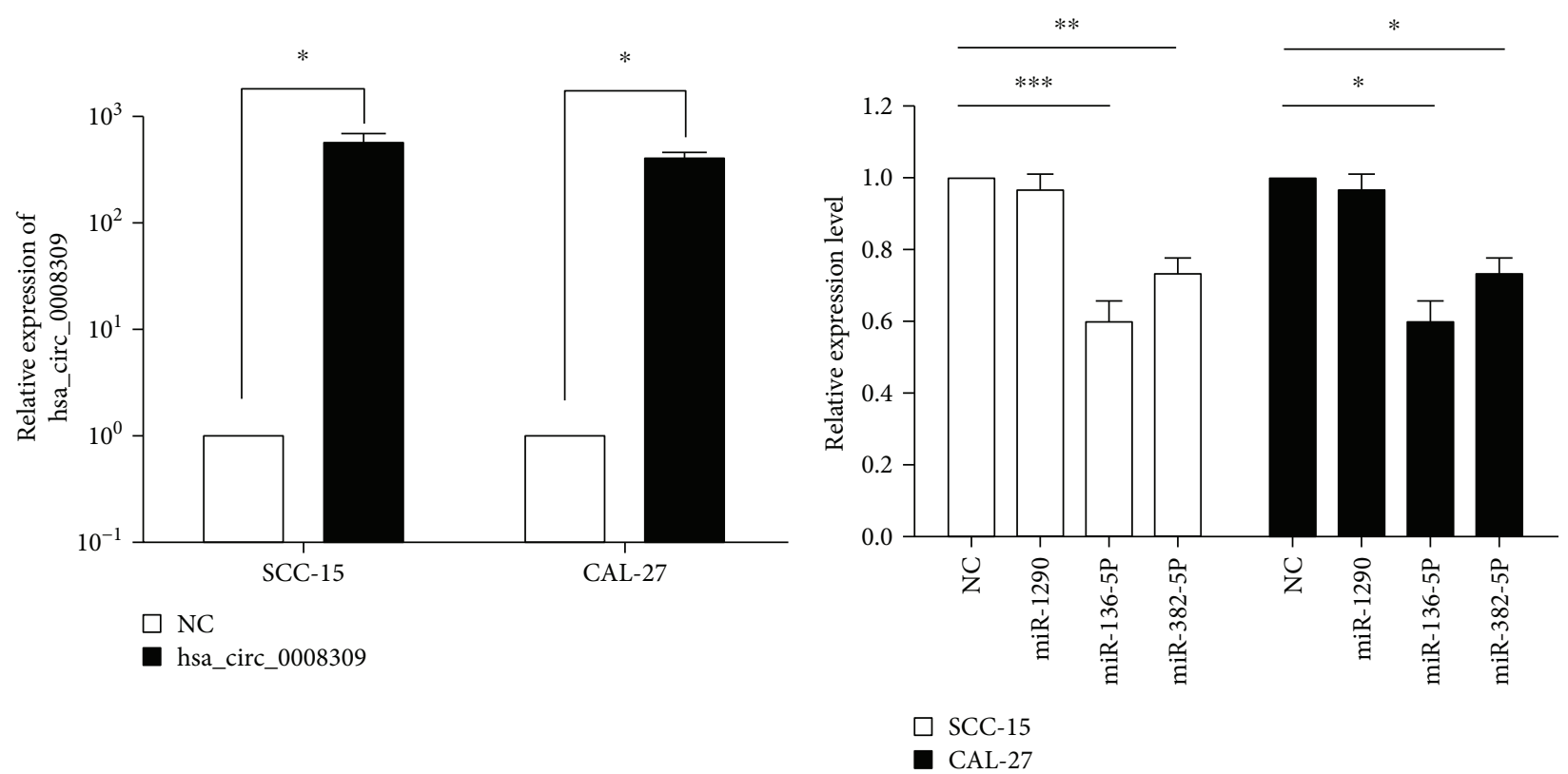

(a)

(b)
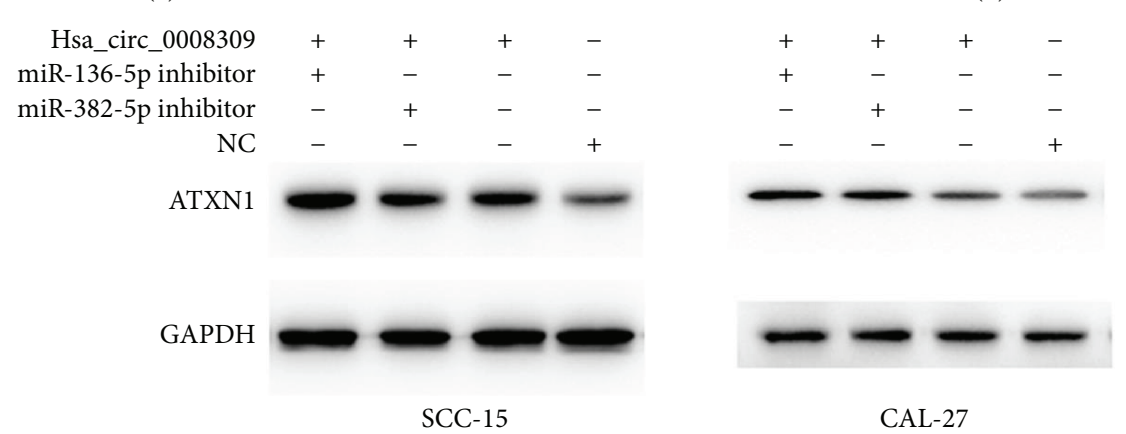

(c)

FIgURE 4: (a) RT-qPCR analysis illustrating the hsa_circ_0008309 expression levels following transfection in SCC15 and CAL27 cell lines. Hsa_circ_0008309 was significantly overexpressed compared with the negative control. A Student's $t$-test (two-tailed) was performed. Data are shown as the mean \pm standard deviation, ${ }^{*} P<0.05$. (b) Overexpression of hsa_circ_0008309 significantly downregulates the expression of miR-136-5P and miR-382-5P compared with control in SCC15 and CAL27 cell lines by RT-qPCR. One-way ANOVA with Tukey's post-hoc test was performed. Data are shown as the mean \pm standard deviation, ${ }^{*} P<0.05,{ }^{* *} P<0.01$. (c) Western blot analysis shows the ATXN1 protein level in SCC15 and CAL27 cell lines following transfection with hsa_circ_0008309 expression plasmid, miR-1365P inhibitor plus hsa_circ_0008309 expression plasmid, miR-382-5P inhibitor plus hsa_circ_0008309 expression plasmid and a mock vector. GAPDH was used as an internal control. RT-qPCR: reverse transcription-quantitative polymerase chain reaction; ATXN1: Ataxin 1; miR: microRNA.

but it remains unknown whether ATXN1 acts as a tumor suppressor or an oncogene. Therefore, the ATXN1 might play an important role in the OSCC. According to bioinformatics analysis, the experimental data obtained by the present study suggest that hsa_circ_0008309, a novel emerging circRNA, may regulate ATXN1 by miR-136-5P and miR382-5P in OSCC cell lines.

In summary, the present study revealed that the OSCC tissues have abundant circRNAs and identified that hsa_circ_0008309 was significantly downregulated in OSCC tissues. Herein is an offered novel approach to explore the role of circRNA by various bioinformatics analyses. Furthermore, the regulatory role of hsa_circ_0008309-miR136-5P/hsa-miR-382-5P-ATXN1 network was identified in
OSCC. These results highlighted the possibility that hsa circ_0008309 could serve as a potential target for OSCC. The functions and mechanisms of hsa_circ_0008309 in OSCC should continue to be extensively investigated.

\section{Data Availability}

The data used to support the findings of this study are available from the corresponding author upon request.

\section{Disclosure}

This paper has not been published elsewhere in whole or in part. All authors have read and approved the content 
and agree to submit for consideration for publication in the journal. There is no any ethical/legal conflicts involved in the article.

\section{Conflicts of Interest}

The authors declare that they have no conflicts of interest.

\section{Authors' Contributions}

Bowen Li and Feng Wang contributed equally to this work.

\section{Acknowledgments}

The paper was supported by the National Natural Science Foundation of China (grant no. 81572654), the Basic Research Program of Shenzhen Innovation Council of China (grant no. JCYJ20150403091443303, JCYJ20150403091443 286, CYJ201604287 3933559, and SZBC2017023), and San Ming Project of Medicine in Shenzhen (SZSM 201512036, Oral and Maxillofacial Surgery Team, Professor GuangYan $\mathrm{Yu}$, Peking University Hospital of Stomatology).

\section{References}

[1] M. S. Rosebush, S. K. Rao, S. Samant et al., "Oral cancer: enduring characteristics and emerging trends," The Journal of the Tennessee Dental Association, vol. 91, no. 2, pp. 24-27, 2011.

[2] J. Casiglia and S. B. Woo, "A comprehensive review of oral cancer," General Dentistry, vol. 49, no. 1, pp. 72-82, 2001.

[3] Z. J. Sun, G. Chen, W. Zhang et al., "Mammalian target of rapamycin pathway promotes tumor-induced angiogenesis in adenoid cystic carcinoma: its suppression by isoliquiritigenin through dual activation of c-Jun NH2-terminal kinase and inhibition of extracellular signal-regulated kinase," The Journal of Pharmacology and Experimental Therapeutics, vol. 334, no. 2, pp. 500-512, 2010.

[4] K. F. He, L. Zhang, C. F. Huang et al., "CD163+ tumorassociated macrophages correlated with poor prognosis and cancer stem cells in oral squamous cell carcinoma," BioMed Research International, vol. 2014, Article ID 838632, 9 pages, 2014.

[5] K. D. Miller, R. L. Siegel, C. C. Lin et al., "Cancer treatment and survivorship statistics, 2016," CA: a Cancer Journal for Clinicians, vol. 66, no. 4, pp. 271-289, 2016.

[6] Y. Li, Q. Zheng, C. Bao et al., "Circular RNA is enriched and stable in exosomes: a promising biomarker for cancer diagnosis," Cell Research, vol. 25, no. 8, pp. 981-984, 2015.

[7] S. Qu, X. Yang, X. Li et al., "Circular RNA: a new star of noncoding RNAs," Cancer Letters, vol. 365, no. 2, pp. 141-148, 2015.

[8] S. Memczak, M. Jens, A. Elefsinioti et al., "Circular RNAs are a large class of animal RNAs with regulatory potency," Nature, vol. 495, no. 7441, pp. 333-338, 2013.

[9] H. Zhang, K. Cai, J. Wang et al., "MiR-7, inhibited indirectly by lincRNA HOTAIR, directly inhibits SETDB1 and reverses the EMT of breast cancer stem cells by downregulating the STAT3 pathway," Stem Cells, vol. 32, no. 11, pp. 2858-2868, 2014.

[10] W. Yang, W. W. Du, X. Li, A. J. Yee, and B. B. Yang, "Foxo3 activity promoted by non-coding effects of circular RNA and
Foxo3 pseudogene in the inhibition of tumor growth and angiogenesis," Oncogene, vol. 35, no. 30, pp. 3919-3931, 2016.

[11] F. Li, L. Zhang, W. Li et al., "Circular RNA ITCH has inhibitory effect on ESCC by suppressing the $\mathrm{Wnt} / \beta$-catenin pathway," Oncotarget, vol. 6, no. 8, pp. 6001-6013, 2015.

[12] S. H. Huang and B. O'Sullivan, "Overview of the 8th edition TNM classification for head and neck cancer," Current Treatment Options in Oncology, vol. 18, no. 7, p. 40, 2017.

[13] P. Glažar, P. Papavasileiou, and N. Rajewsky, "circBase: a database for circular RNAs,” RNA, vol. 20, no. 11, pp. 1666-1670, 2014.

[14] G. J. Spector, R. H. Ciralsky, and J. H. Ogura, "Glomus tumors in the head and neck: III. Analysis of clinical manifestations," Annals of Otology, Rhinology \& Laryngology, vol. 84, no. 1, pp. 73-79, 1975.

[15] Z. Zhong, M. Lv, and J. Chen, "Screening differential circular RNA expression profiles reveals the regulatory role of circTCF25-miR-103a-3p/miR-107-CDK6 pathway in bladder carcinoma," Scientific Reports, vol. 6, no. 1, p. 30919, 2016.

[16] H. L. Sanger, G. Klotz, D. Riesner, H. J. Gross, and A. K. Kleinschmidt, "Viroids are single-stranded covalently closed circular RNA molecules existing as highly base-paired rodlike structures," Proceedings of the National Academy of Sciences of the United States of America, vol. 73, no. 11, pp. 3852-3856, 1976.

[17] Z. Li, C. Huang, C. Bao et al., "Exon-intron circular RNAs regulate transcription in the nucleus," Nature Structural \& Molecular Biology, vol. 22, no. 3, pp. 256-264, 2015.

[18] M. T. Hsu and M. Coca-Prados, "Electron microscopic evidence for the circular form of RNA in the cytoplasm of eukaryotic cells," Nature, vol. 280, no. 5720, pp. 339-340, 1979.

[19] T. B. Hansen, T. I. Jensen, B. H. Clausen et al., "Natural RNA circles function as efficient microRNA sponges," Nature, vol. 495, no. 7441, pp. 384-388, 2013.

[20] J. Li, J. Yang, P. Zhou et al., "Circular RNAs in cancer: novel insights into origins, properties, functions and implications," American Journal of Cancer Research, vol. 5, no. 2, pp. 472480, 2015.

[21] I. A. Klement, P. J. Skinner, M. D. Kaytor et al., "Ataxin-1 nuclear localization and aggregation: role in polyglutamineinduced disease in SCA1 transgenic mice," Cell, vol. 95, no. 1, pp. 41-53, 1998.

[22] X. Tong, H. Gui, F. Jin et al., "Ataxin-1 and brother of ataxin-1 are components of the Notch signalling pathway," EMBO Reports, vol. 12, no. 5, pp. 428-435, 2011.

[23] C. Sahlgren, M. V. Gustafsson, S. Jin, L. Poellinger, and U. Lendahl, "Notch signaling mediates hypoxia-induced tumor cell migration and invasion," Proceedings of the National Academy of Sciences of the United States of America, vol. 105, no. 17, pp. 6392-6397, 2008.

[24] A. R. Kang, H. T. An, J. Ko, E. J. Choi, and S. Kang, "Ataxin-1 is involved in tumorigenesis of cervical cancer cells via the EGFR-RAS-MAPK signaling pathway," Oncotarget, vol. 8, no. 55, pp. 94606-94618, 2017.

[25] A. R. Kang, H. T. An, J. Ko, and S. Kang, "Ataxin-1 regulates epithelial-mesenchymal transition of cervical cancer cells," Oncotarget, vol. 8, no. 11, pp. 18248-18259, 2017. 


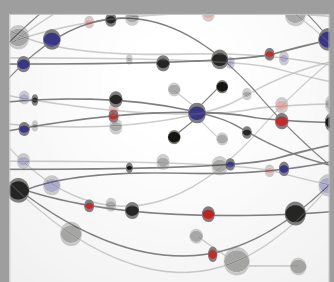

The Scientific World Journal
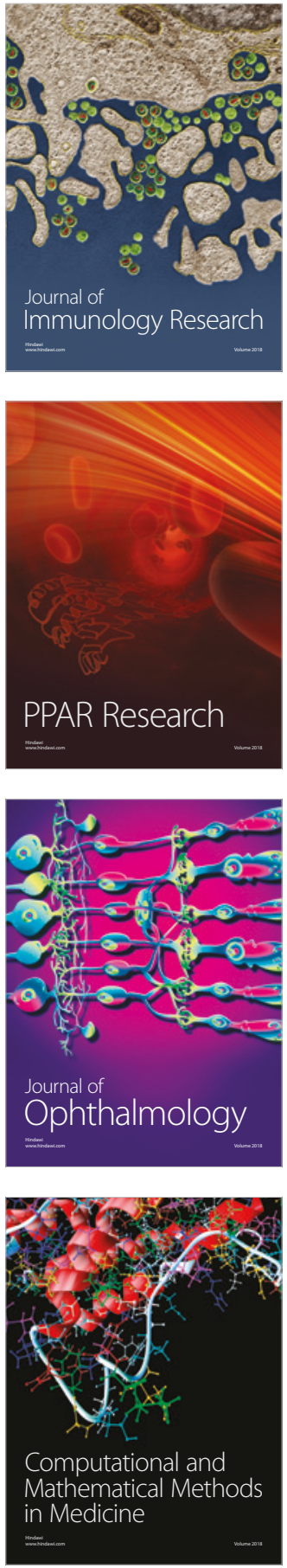

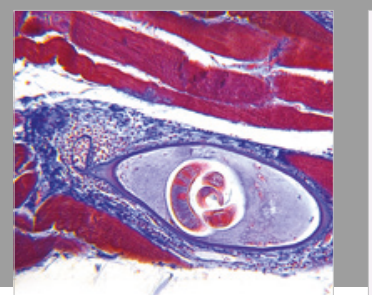

Gastroenterology Research and Practice

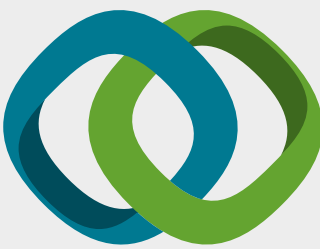

\section{Hindawi}

Submit your manuscripts at

www.hindawi.com
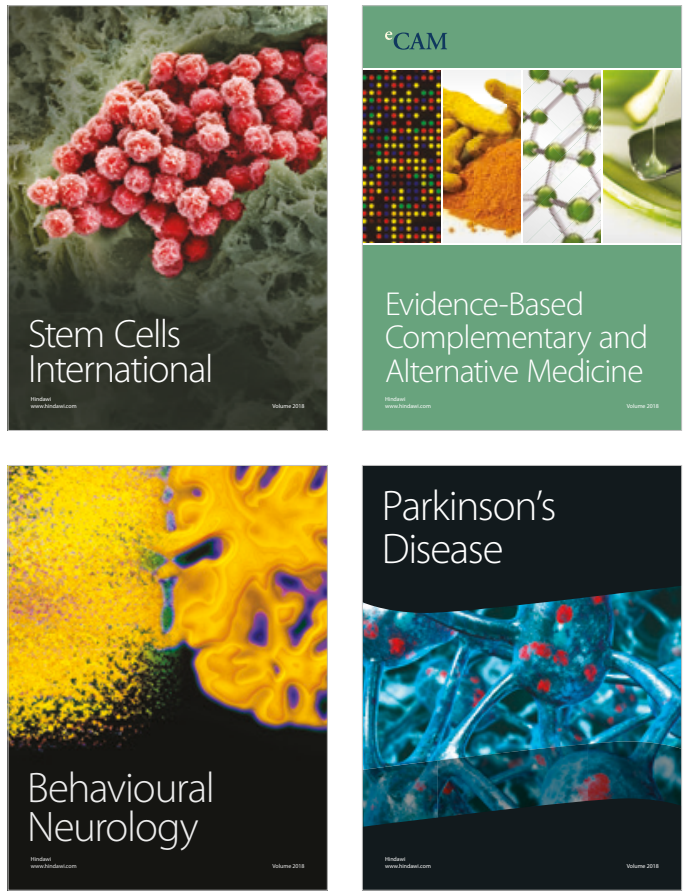

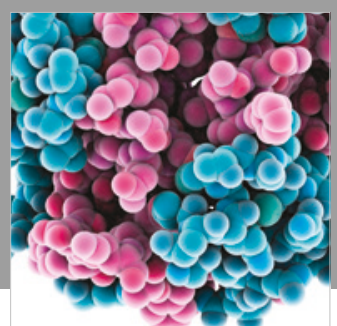

ournal of

Diabetes Research

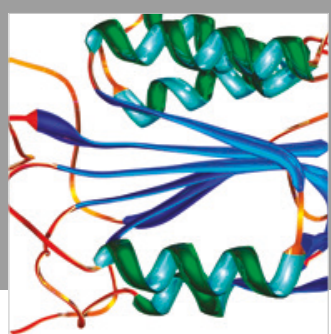

Disease Markers
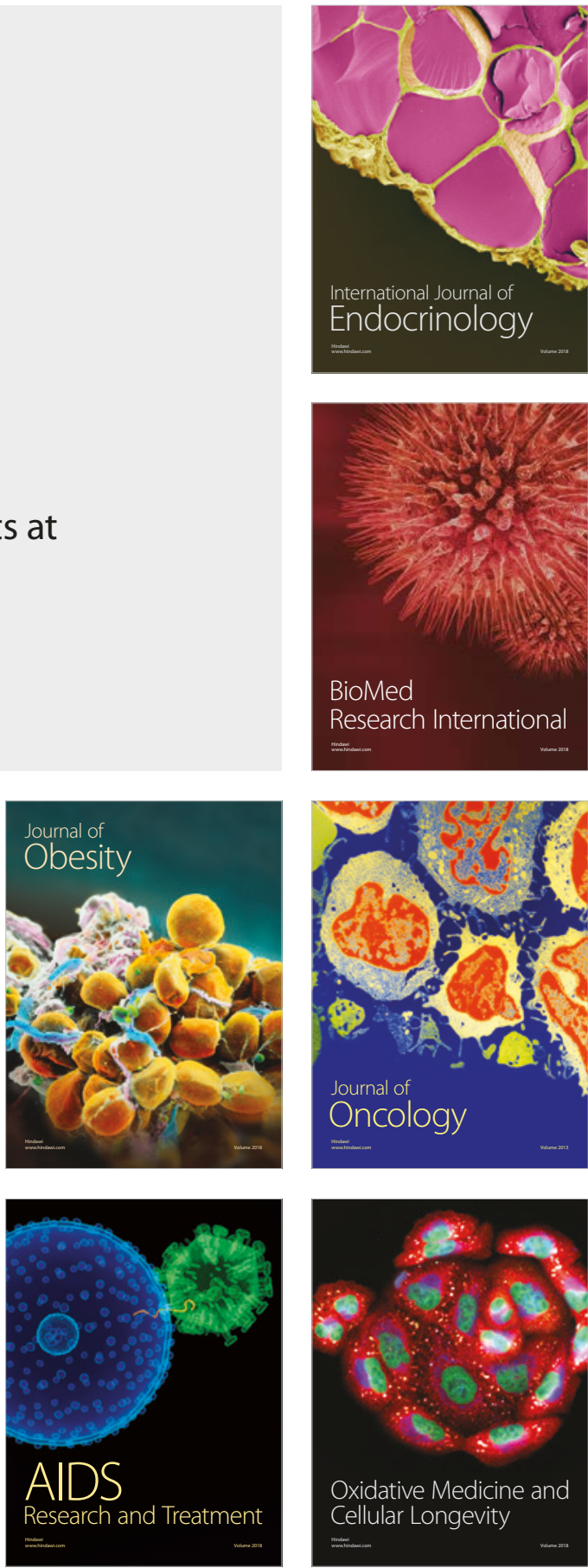